\title{
PATHOLOGICAL MONITORING OF VISCERAL LESIONS INDUCED BY VELOGENIC NEWCASTLE DISEASE IN EXPERIMENTALLY INFECTED BROILER CHICKENS AT DIFFERENT AGES WITH SPECIAL REFERENCE TO PULMONARY INFLAMMATORY CYTOKINES EXPRESSION
}

\author{
Mohamed R. Mousa ${ }^{1}$, Faten F. Mohammed ${ }^{1}$, Ayman H. El-Deeb², Fatma Amer ${ }^{3}$, Kawkab A. Ahmed ${ }^{1}$ \\ Received 21 January 2021, revised 29 May 2021
}

\begin{abstract}
The present work aimed to study the impact of infection with a locally isolated vNDV genotype VII in commercial broiler chickens of different ages from a histopathological point of view with related changes in pulmonary cytokine expression. 100 commercial broiler chickens were allocated into 4 groups as follows; group 1 served as control non-infected group and three infected groups, were infected with 106 EID50 NDV at ages of 10,20, and 30 days. Tissue samples from different organs were collected at the 1st, 3rd, 5th, and 7th days post-inoculation for histopathological and immunohistochemical examination. Quantitative real-time RT-PCR for virus re-isolation as well as expression of IL-6 and IFN- $\gamma$ genes estimation were performed on lung tissue collected at 3 dpi. RT-PCR for virus revealed increased virus load in the lungs of older birds and cytokines profiling revealed significant up-regulation of both IL-6 and IFN- $\gamma$ genes in the groups that received infection at older ages. The severity and distribution of pathological lesions were directly proportioned to the age of infection and the time interval post-infection. Immunostaining of the viral antigen in the different tissues confirmed the pantropic nature of the virus.
\end{abstract}

Key words: Velogenic New Castle Disease virus, IL-6, IFN- $\gamma$, Pathology, Immunohistochemistry.

\section{INTRODUCTION}

Newcastle disease (ND) is a highly contagious viral disease of poultry and has a devastating effect on poultry production (Chukwudi et al. 2012). ND is responsible for high morbidity and mortality in susceptible birds of all age groups (Siddique et al. 2012, Ahmed and Odisho 2018).

In developing countries where the majority of chickens are reared under "backyard" conditions, ND can drastically limit the amount of dietary protein as well as damage the micro-economy by decreasing chickens' meat or egg production (Cattoli et al. 2011). ND virus (NDV) is an enveloped, single-stranded, negative-sense RNA virus classified within the family Paramyxoviridae with isolates designated as avian paramyxovirus-1 (APMV1) (Mayo 2002).
Velogenic isolates are further divided into a velogenic viscerotropic pathotype, which are those strains causing predominantly diarrhea and visceral hemorrhage, and a velogenic neurotropic pathotype, which are those strains causing predominantly neurologic signs (Hanson et al. 1973).

The velogenic viscerotropic Newcastle disease (vvND) is a common type of ND that occurs in many parts of the world. It is caused by virulent strains of the genus Avulavirus and the family Paramyxoviridae (World Organisation for Animal Health) (OIE 2012).

The velogenic pathotype of ND is the most severe form of ND with the velogenic viscerotropic pathotype being the most common and enzootic in Africa, the Middle and the Far East, and some countries of Central and South America (Iroegbu and Emeruwa 1993, Liu et al. 2003).

\footnotetext{
${ }^{1}$ Department of Pathology, ${ }^{2}$ Department of Virology, Faculty of Veterinary Medicine, Cairo University, Giza, 12211, Egypt.

${ }^{3}$ Reference Laboratory for Veterinary Quality Control on Poultry Production (RLQP), Animal Health Research Institute (AHRI), Giza, Egypt.

*Corresponding author. e-mail: fatenfathy21@yahoo.com.
} 
NDV exhibits differences in tropism and virulence depending on the activation of a viral fusion protein precursor by cellular proteases (Gotoh et al. 1992, Nagai 1995).

Genotype VII NDV induced systemic infection with prominent tropism in visceral organs in chicken was confirmed based on gross and microscopic lesions, and immunohistochemistry findings (Desingu et al. 2017).

The main histologic lesions of ND are nonpurulent encephalomyelitis (Mohammed et al. 2019), vasculitis, lymphoid necrosis (cloacal bursa, spleen, thymus, and intestinal mucosal lymphoid tissue), tracheitis and pneumonia, salpingitis, liver necrosis, cellular infiltration of the pancreas, and conjunctivitis (Alexander 2003).

Matting of vent feathers, petechial haemorrhage on the tip of proventriculus papilla, ventriculus, intestine and caecal tonsil, congestion of trachea and lung were also reported as most commonly detected lesions due to vvNDV infection (Xiao et al. 2012, Panus et al. 2015, Onyema et al. 2019). VVND infection also had been reported to cause multifocal areas of necrosis in the pancreas, liver, and gall bladder (Kommers et al. 2002, Kommers et al. 2003, Susta et al. 2011).

Our experimental work aimed to investigate the pathological characterization of lesions developed in different visceral organs induced by experimental infection with vvNDV in relation to the age of infected chickens, immunohistochemical detection of viral antigen in different organs, and characterization of pulmonary cytokines expression.

\section{MATERIALS AND METHODS}

\section{Virus}

Local strain of velogenic NDV genotype VII (NDVB7-RLQP-CH-EG-12) isolated from Kafr El-sheikh governorate, Egypt was provided by National Laboratory for Veterinary Control on Poultry Production, Animal Health Research Institute. The partial sequencing of $F$ gene was deposited in Gene Bank under accession number KM288609. Determination of the egg infectious dose 50 (EID50) was performed according to Reed and Muench (1938) and 106 EID50 was used for infection of the chickens.

\section{Experimental design}

One hundred day-old Ross commercial broiler chicks were kept in separate rooms and were provided with water and feed ad libitum. Routine vaccination against avian influenza H5N1 (Inactivated H5N1, S/C, $0.5 \mathrm{ml} /$ bird) and infectious bursal disease (IBD 228-E, intraocular, one drop/bird) virus at 10 days old were performed and no vaccination for NDV was used. The birds were randomly allocated into four groups ( 25 per each) as follows; group 1 (control non-infected), group 2 (infected at 10 days old), group 3 (infected at 20 days old), and group 4 (infected at 30 days). The infected groups were inoculated intraocular with a dose of $106 \mathrm{EID}_{50} \mathrm{NDV}$ (in a total volume of $0.1 \mathrm{ml}$ ). Three chickens from each group were sacrificed at 1-, 3-, 5-, and 7-days post-inoculation (dpi). On each sacrifice, trachea, lungs, proventriculus, liver, pancreas, and heart tissue samples were collected from all experimental groups. This experimental protocol was approved by Institutional Animal Care and Use Committee (IACUC), Cairo University, Egypt (Approval number, CU/II/F/65/17).

\section{Real time RT-PCR for detection of virulent ND}

Specific primers and probes designed by Wise et al. (2004) were supplied from Metabion (Germany) with a PCR kit from (Qiagen, Inc. Valencia CA, Cat. no. 204443) were used.

Primers were (F+4839) 5'- TCC GGA GGATAC AAG GGT CT-3', (F-4939) 5'- AGC TGT TGC AAC CCC AAG-3' and probe was $(\mathrm{F}+4894)$ 5'- [FAM] AAG CGT TTC TGT CTC CTT CCT CCA[TAMRA] -3'.

After RNA extraction, NDV specific primers were utilized in a $25 \mu \mathrm{l}$ reaction containing $12.5 \mu \mathrm{l}$ of Quantitect probe rt-PCR master mix (Qiagen, Germany, GmbH), $0.5 \mu \mathrm{l}$ of each primer of $50 \mathrm{pmol}$ concentration, $0.125 \mu \mathrm{l}$ of the specific probe, $4.125 \mu \mathrm{l}$ of RNase Free Water, $0.25 \mu \mathrm{l}$ of QuantiTect RT Mix and $7 \mu$ l of RNA extract. The reaction was performed in a Stratagene MX3005P real-time PCR machine (Stratagene, USA). The reserve transcription step was performed at $50^{\circ} \mathrm{C}$ for $30 \mathrm{~min}$ followed by $15 \mathrm{~min}$ at $95^{\circ} \mathrm{C}$ as primary denaturation, while the cycle protocol were 40 cycles of three successive steps; $94^{\circ} \mathrm{C}$ for $30 \mathrm{sec}, 52^{\circ} \mathrm{C}$ for $30 \mathrm{sec}$ and $72^{\circ} \mathrm{C}$ for $10 \mathrm{sec}$.

\section{Cytokine gene expression}

Lung tissues collected at 3 days post-infection (dpi) were used for extraction of the total RNA using a RNeasy Mini RNA Purification Kit and RNase-Free DNase Kit (QIAGEN). The expression of different cytokines was quantified by reverse transcription real-time PCR (RTPCR) using relative quantification. The primers and probes used for the determination of IL-6, IFN- $\gamma$, and 28srRNA were previously mentioned by Kaiser et al. (2000).

Quantitative real-time RT-PCR was performed using Quantitect probe RT-PCR (QIAGEN) according to the manufacturer's recommendations. Real-time RT-PCR 


\section{Cytokines expression}

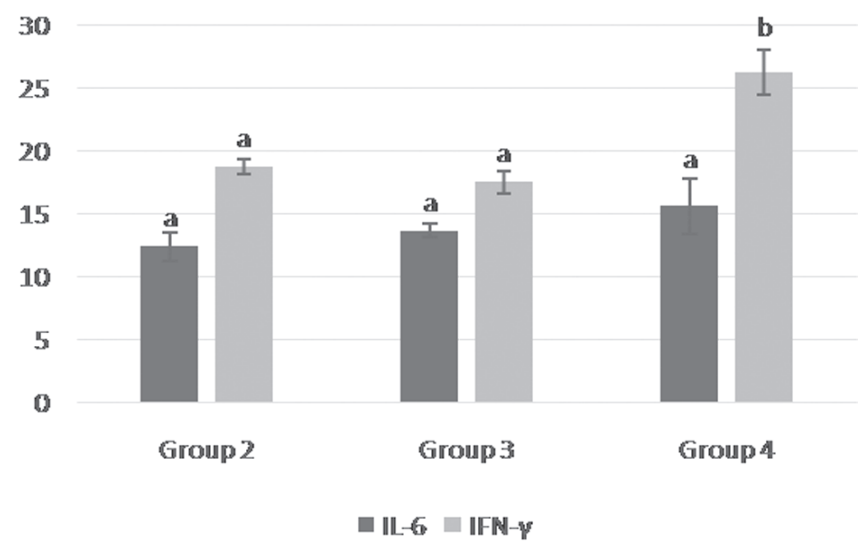

Fig. 1. Relative expression of cytokines in the lungs of infected chickens from different infected groups for IL-6 (black column) and IFN- $\boldsymbol{\gamma}$ (gray column).

[Letters $(a-b)$ on the bars indicate statistically significant differences at $\mathrm{p}=0.05]$.

was carried out using a 7500 Real-time PCR System (Applied Biosystems). PCR conditions were the same for each cytokine gene, as follows: $30 \mathrm{~min}$ at $50^{\circ} \mathrm{C}, 95^{\circ} \mathrm{C}$ for $15 \mathrm{~min}$, followed by 40 cycles of $95^{\circ} \mathrm{C}$ for 15 sand $60^{\circ} \mathrm{C}$ for $1 \mathrm{~min}$.

Statistical analysis for fold changes in cytokine levels was determined by the $\Delta \Delta \mathrm{Ct}$ method (Livak and Schmittgen 2001), using 28S ribosomal RNA as the endogenous reference gene to normalize the level of the target gene expression.

\section{Clinical findings and mortality rate}

Chickens in all experimental groups were daily monitored for abnormal clinical signs and mortality which were recorded in all groups.

\section{Gross, histopathological and immunohistochemical investigations}

On dissection, all visceral organs were thoroughly examined to detect any macroscopic abnormalities. Trachea, lungs, proventriculus, liver, pancreas, and heart tissue samples were collected and kept in $10 \%$ neutral buffered formalin solution, routinely processed, sectioned at $5 \mu \mathrm{m}$ thickness, and stained with Hematoxylin and Eosin (H\&E) for subsequent histopathological examination (Bancroft 2013). Tissue slides were examined by Olympus BX43 light microscope and captured using Olympus DP27 camera linked to Cellsens dimensions software (Olympus).

For immunohistochemistry, hyperimmune serum against NDV was raised in rabbits using series of injections following the schedule described by Samiullah et al. (2006). Antibody purification was performed using Magne $^{\mathrm{TM}}$ Protein G Beads for Antibody Purification according to the manufacturer's instructions. Tissue sections from paraffin blocks at 5 and 7 dpi were sectioned on Poly-L-Lysine coated slides, then they were deparaffinized and rehydrated, as usual, the antigen retrieval step was performed by heat induction, blocking of non-specific protein binding and endogenous peroxide was followed by overnight incubation in primary antibody (Rabbit anti-NDV Ig previously mentioned) then incubated with horseradish peroxidase-conjugated goat polyclonal secondary antibody to rabbit Ig (SM802 EnVision ${ }^{\mathrm{TM}}$ FLEX/HRP). The color was developed with 3, 32 -Diaminobenzidine (DAB) substrate (DM827 EnVision ${ }^{\mathrm{TM}}$ FLEX DAB + Chromogen) and counterstained with Mayer's hematoxylin. For negative control, the primary antibody was replaced by phosphatebuffered saline (Burns et al. 2005).

\section{Histopathological lesions score}

The scoring system for the detected microscopic findings in each organ was done according to Hussein et al. (2018) with some modifications. It was as follows: Trachea: $0=$ normal; $1=$ hyperemia and inflammatory cells infiltration; 2 = hyperemia, inflammatory cells infiltration, and edema; 3 = hyperemia, inflammatory cells infiltration, edema and deciliation; 4 =slight hyperplasia and deciliation; $5=$ hemorrhagic patches, desquamation and hyperplasia.

Lungs: $0=$ normal; $1=$ inflammatory cells infiltration in the air capillaries; $2=$ inflammatory cells infiltration, hemorrhage, and exudate into the secondary bronchi; 3 $=$ hypertrophy of the tertiary bronchial epithelium and interstitial edema.

Proventriculus: $0=$ normal; $1=$ mild epithelial cell degeneration and necrosis with heterophils; $2=$ extensive epithelial cell degeneration and necrosis accompanied by mononuclear cell infiltration; $3=$ destruction of the lymphoid areas and often fibrin was present; 4 = destroyed lymphoid areas with some hemorrhage.

Liver: $0=$ normal; $1=$ inflammatory cells infiltration; 2 = inflammatory cells infiltration accompanied by hepatocellular degeneration and necrosis; $3=$ severe hepatocellular degeneration and necrosis.

Pancreas: $0=$ normal; $1=$ degeneration of the exocrine glandular acini and blood vessels congestion; $2=$ apoptosis of the exocrine glandular acini; $3=$ inflammatory cells infiltration; $4=$ inflammatory cells infiltration with severe destruction of the pancreatic parenchyma. 

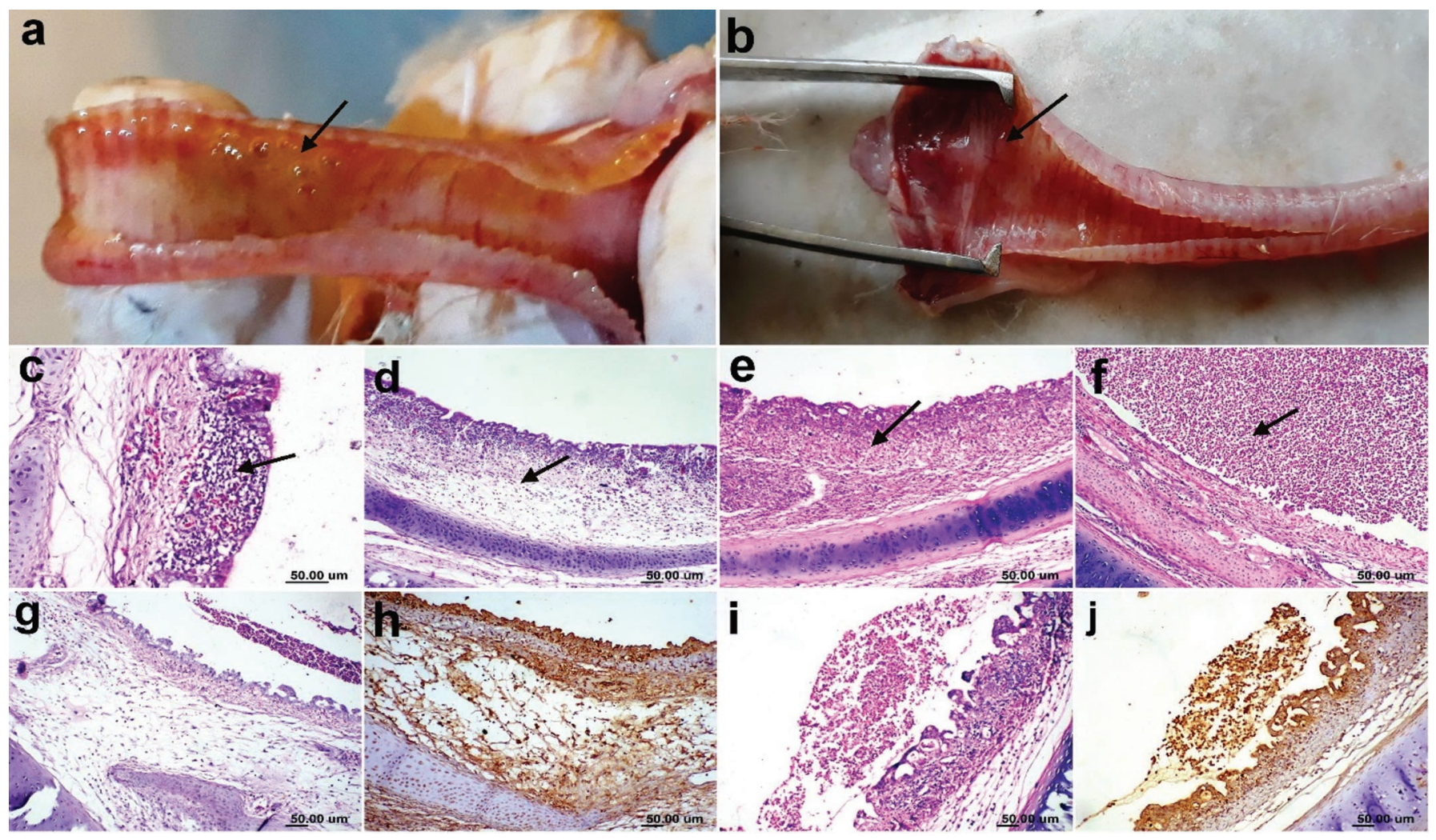

Fig. 2. Pathological findings of the trachea of NDV infected chicken.

[(a) Group 3 at 5 dpi showing catarrhal tracheitis with heavily mucous exudation in the tracheal lumen (arrow). (b) Group 4 at 5 dpi presenting severe hemorrhagic tracheitis (arrow). (c) Group 2 at 1 dpi showing focal deciliation with a focal inflammatory infiltrate (arrow) (H\&E). (d) Group 3 at 1 dpi showing severe deciliation, submucosal edema with mononuclear cells infiltration (arrow) (H\&E). (e) Group 3 at 3 dpi showing severe tracheitis with mononuclear cells infiltration (arrow)and reduction of the mucous glands (H\&E). (f) Group 3 at 7 dpi showing severe massive exudation of heterophils in the tracheal lumen (arrow) (H\&E). (g) Group 4 at 5 dpi showing submucosal inflammatory edema (arrow) with destructed mucosal surface (H\&E). (h) Group 4 at 5 dpi showing expression of viral antigen in the destructed mucosal layer and the edematous submucosal layer (immunohistochemistry (IHC). (i) Group 4 at 7 dpi showing heterophilic infiltration in the tracheal lumen (H\&E) and (j) Group 4 at $7 \mathrm{dpi}$ showing expression of the viral antigen in the heterophils exuding the tracheal lumen and the necrosed tissue (IHC)].

Heart: $0=$ normal; $1=$ infiltration of mononuclear inflammatory cells; 2 = degeneration of myocardial fibers; $3=$ myocardial fibers started to disrupt; $4=$ disruption of cardiac myofibers and macrophages accumulation in the myocardium.

\section{Statistical analysis}

Statistical analyses were performed using one-way factorial analysis of variance (ANOVA). Statistical significance was defined as $(\mathrm{p}=0.05)$ using SPSS 17 .

\section{RESULTS AND DISCUSSION \\ Real-Time PCR and cytokine mRNA gene expression}

The PCR result confirmed the highest detection for vND in lung tissues which was collected at 3 dpi in group 4 (30 days old) in comparison with group 3 (20 days old) and group 2 (10 days old).

Although there were no significant differences among all infected groups, group 4 (30 days age infected group) recorded the highest expression of IL-6at 3 dpi compared with groups 2 and 3 (10 and 20 days old respectively). Meanwhile, no significant difference in gene expression of IFN- $\gamma$ was detected in infected chickens of 10 and 20 days old compared to the group of 30 days old that conversely showed a significantly higher expression of $\operatorname{IFN} \gamma$ (Fig. 1).

\section{Clinical signs and mortality rate}

The control group showed no abnormal clinical signs, with health signs that were manifested by increased feed and water consumption with normal vitality and no recorded mortality during the experimental period. However, group 2 (infected at 10 days old) showed respiratory sounds at $6 \mathrm{dpi}$ in addition to few birds exhibited greenish diarrhoea at $7 \mathrm{dpi}$. No mortalities were observed in this group. On the other hand, birds in group 3 showed depression and greenish diarrhoea ( $5 \mathrm{dpi}$ ). Signs 
Pathological monitoring of visceral lesions induced by velogenic newcastle disease...

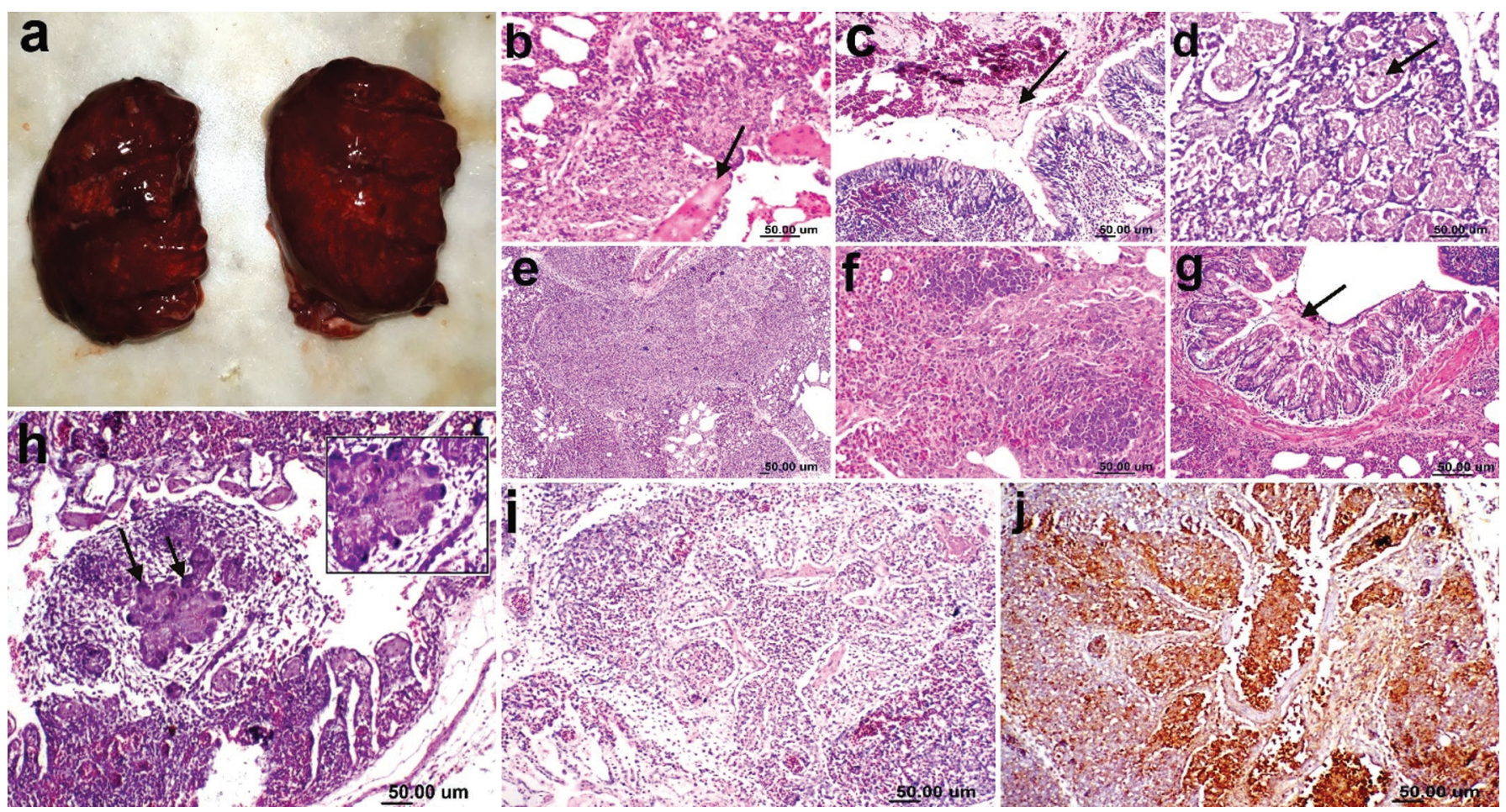

Fig. 3. Pathological findings of the lung of NDV infected chicken.

[(a) Group 4 at 7 dpi showing severe lung congestion, (b) Group 2 at 3 dpi showing proliferative response of parabronchial wall with hypertrophy of the smooth muscle (arrow) (H\&E). (c) Group 3 at 3 dpi showing activation of the goblet cells in the mucosal surface of secondary bronchi with mucous exudate in the lumen mixed with few mononuclear cells (arrow) (H\&E). (d) Group 3 at 7 dpi showing lung edema with occluding of the air spaces by eosinophilic proteinous material (arrow) (H\&E). (e) Group 4 at 5 dpi showing severe lung consolidation by massive mononuclear cells infiltration (H\&E).(f) Group 4 at 7 dpi showing air capillaries infiltrated with mononuclear cells(H\&E). (g) Group 4 at 7 dpi showing folding of mucosal surface lining the secondary bronchi with hyperplasia of the goblet cells and mucous exudate covering the mucosa (arrow)(H\&E). (h) Group 4 at 7 dpi showing the formation of syncytial cells (arrows) (insert) mixed with mononuclear cells in the lumen of the parabronchus (H\&E). (i) Group 4 at 7 dpi showing occlusion of the para-bronchus lumen by inflammatory reaction, atria and infundibula with massive mononuclear and heterophilic cells infiltration (H\&E). (j) Group 4 at 7 dpi showing expression of the viral antigen in the inflammatory cells (IHC)].

were more severe at $6 \mathrm{dpi}$ as birds appeared severely depressed, lethargic with ruffled feathers, drop in feed consumption with high respiratory sounds. $48 \%$ of infected birds showed nervous manifestation that was evident at 7 dpi in which birds showed curled leg, reluctantance to move, head droop toward the ground and the bird gasping for air as it tried to clear the oral cavity of fluid, extension of legs and head or muscular tremors, torticollis, and paralysis. Total mortality recorded in this group was $8 \%$.

Group 4 developed depression, ruffled feathers with respiratory signs at $1 \mathrm{dpi}$. A decrease in feed intake was noticed from 2 dpi, while loss of appetite was observed starting from 3 dpi admixed with greenish diarrhoea. 20 $\%$ of infected birds showed nervous manifestation that was noticed at $7 \mathrm{dpi}$ with total mortality of $24 \%$.

\section{Pathological findings \\ Trachea}

Grossly, group 2 showed mild tracheal congestion at 7 dpi with no detectable macroscopic findings in the previous sacrifices, conversely, catarrhal tracheitis was observed in group 3 from 5 to $7 \mathrm{dpi}$ with heavily mucous exudation in the tracheal lumen (Fig. 2a), meanwhile, at the same sacrifices group 4 exhibited severe haemorrhagic tracheitis (Fig. 2b) with no detectable gross lesions at 1 and 3 dpi for groups 3 and 4 .

The microscopic examination of group 1 revealed the normal histological structure which was lined by pseudostratified columnar ciliated epithelium with numerous mucous glands. At 1 dpi, group 2 showed focal deciliation with a focal inflammatory area (Fig. 2c). Meanwhile, groups 3 and 4 presented severe deciliation, inflammation with haemorrhage, and edema extending from the submucosal layer into the underlying cartilage and muscular layer (Fig. 2d). At 3 and $5 \mathrm{dpi}$, group 2 showed a mild reduction of the mucous glands with excessive deciliation and submucosal edema. On the contrary, groups 3 and 4 exhibited severe tracheitis which was characterized by mononuclear cells infiltration with 
Exploratory Animal and Medical Research, Vol.11, Issue 1, June, 2021
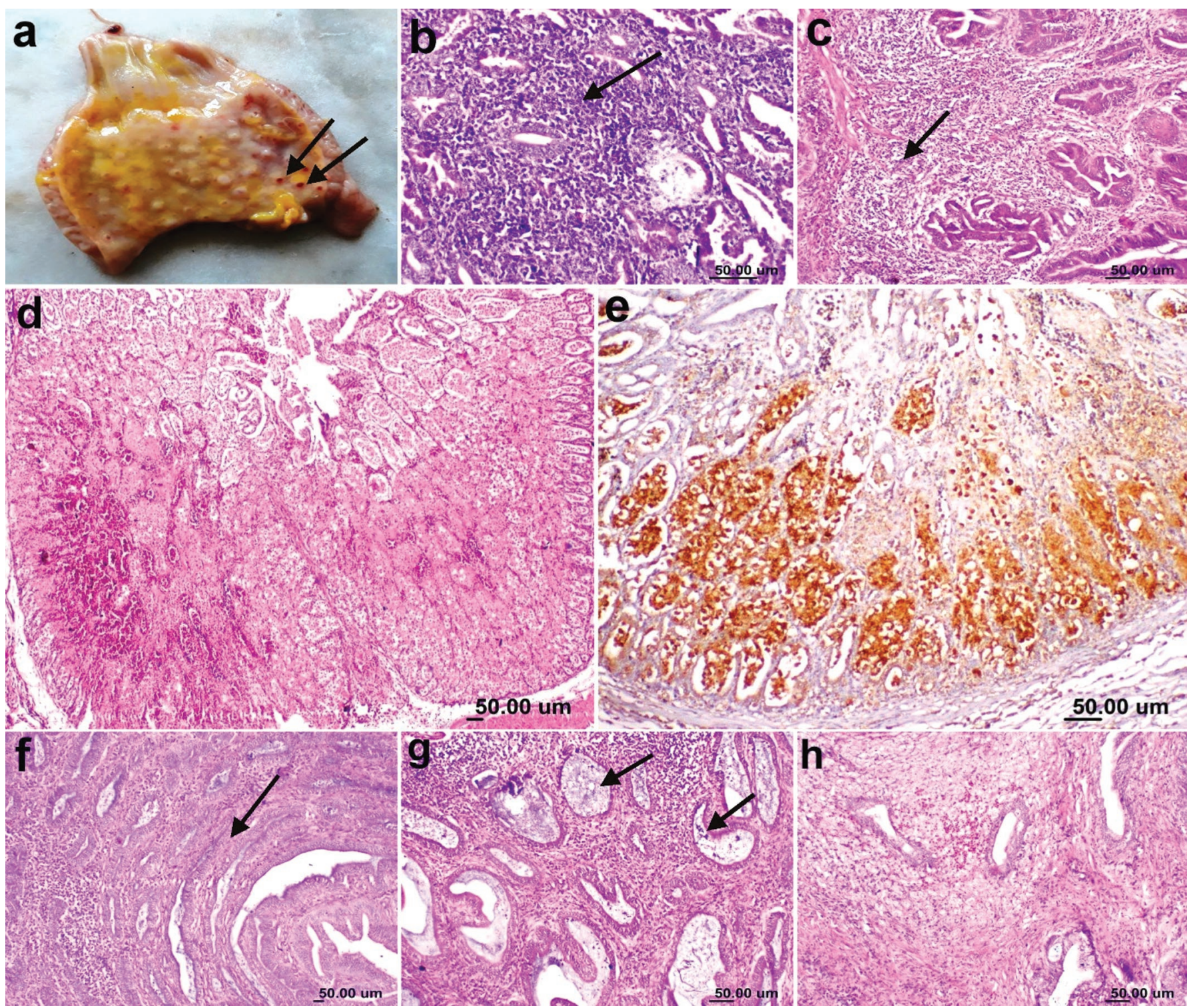

Fig. 4. Pathological findings of the Proventriculus of NDV infected chicken.

[(a) Group 4 at 5 dpi showing petechial hemorrhages on the tips of the proventriculus glands (arrows). (b) Group 2at 5 dpi presenting mononuclear cells infiltrating the necrosed glandular acini (arrow) (H\&E). (c) Group 3 at 3 dpi showing diffuse lymphoplasmacytic infiltration in the submucosa (arrow) (H\&E). (d) Group 4 at 5 dpi showing severe necrohemorrhagic proventriculitis (H\&E).(e) Group 4 at 5 dpi showing numerous expression of the viral antigen in the necrosed glands(IHC).(f) Group 3 at 7 dpi showing fibroplasia of the lamina propria in the mucosal layer (arrow)(H\&E).(g) Group 4 at 7 dpi showing catarrhal proventriculitis with activation of mucous glands (arrows) (H\&E).(h) Group 4 at 7 dpi showing severe depletion of the esophageal tonsil located in the lamina propria of the esophagus at the junction with the proventriculus (arrow) (H\&E)].

heavily reduced mucous glands and vacuolation of the mucosal epithelium (Fig. 2e). Additionally, group 4 revealed the highest degree of tracheal alterations among different infected groups, in which severe tracheitis was observed with mucosal ulceration and massive exudation of heterophilic inflammatory cells in the tracheal lumen (Fig. 2f).

Viral antigen expression was detected in all infected groups from 5 to $7 \mathrm{dpi}$ and was heavily expressed in the damaged mucosal layer with a severe edematous submucosal layer at 5 dpi in group 4 (Fig. $2 \mathrm{~g}$ and $2 \mathrm{~h}$ ).
Moreover, viral antigen was detected in the heterophils infiltrating the tracheal lumen of group 4 at $7 \mathrm{dpi}$ (Fig. $2 \mathrm{i}$ and $2 \mathrm{j}$ ).

\section{Lungs}

No gross lesions were detected in all infected groups from 1 to 7 dpi except severely congested lungs was demonstrated in some birds from group 4 at 7 dpi (Fig. 3a).

Microscopically, group 1 exhibited normal respiratory lobules composed of air and blood capillaries that 
Pathological monitoring of visceral lesions induced by velogenic newcastle disease...
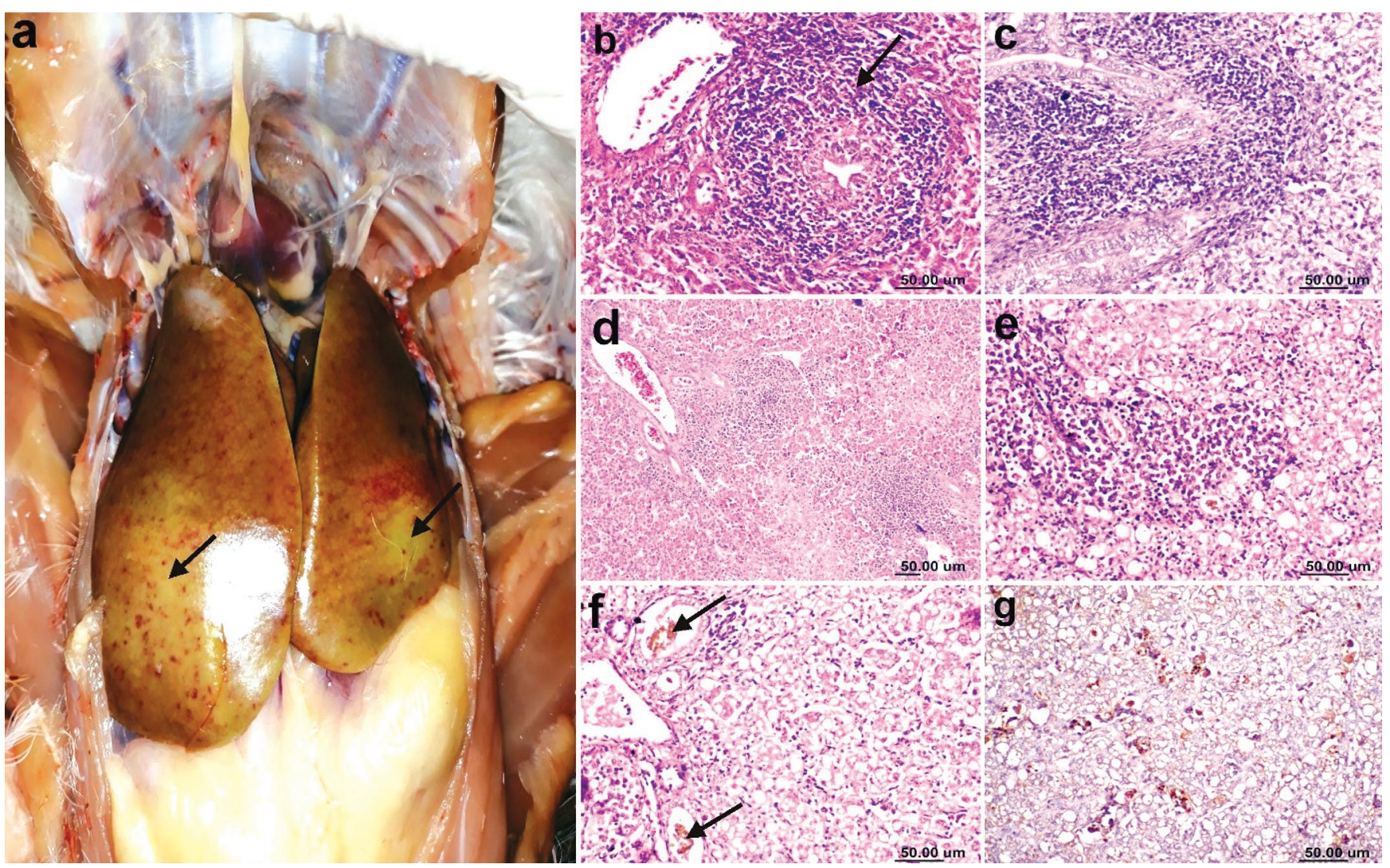

Fig. 5. Pathological findings of the liver of NDV infected chicken.

[(a) Group 4 at 5 dpi showing greenish bronze discoloration of the liver (arrows). (b) Group 3at 3 dpi presenting portal hepatitis with mononuclear cells infiltration (arrow) (H\&E).(c) Group 3 at 5 dpi showing portal hepatitis with adjacent diffuse vacuolation of the hepatocytes(H\&E). (d) Group 3 at 7 dpi showing diffuse hepatitis with heterophils infiltration associated with massive hepatocellular necrosis (H\&E). (e) Group 4 at 5 dpi showing diffuse hepatic necrosis with mononuclear cells infiltration and cholestasis (H\&E). (f) Group 4 at 5 dpi showing cholestasis with the accumulation of bile secretion in the bile ductules (arrows) adjacent to the necrosed hepatic parenchyma (H\&E). (g) Group 4 at 5 dpi showing viral antigen expression in the mononuclear cells infiltrating necrosed hepatic tissue (IHC)].

surround parabronchi. From 1 to 3 dpi, all infected groups showed interstitial edema with variable severity, perivascular edema, and congested blood vessels. Numerous parabronchi of the infected groups showed proliferative response which was characterized by hypertrophy of the smooth muscles and increased cellularity due to hyperplasia and metaplasia (from simple squamous to cuboidal cells) of the epithelium lining the parabronchus, atria, and infundibula with infiltrating lympho-histiocytic cells (Fig. 3b). Moreover, groups 3 and 4 exhibited activation of the goblet cells lining the secondary bronchi with mild to moderate catarrhal bronchitis (Fig. 3c) with submucosal edema and haemorrhage. At $5 \mathrm{dpi}$, group 4 showed eosinophilic proteinaceous exudate flooding the air spaces (Fig. 3d), with massive mononuclear cells infiltration resulting in lung consolidation (Fig. 3e).

At $7 \mathrm{dpi}$, group 3 showed numerous air capillaries infiltrated with mononuclear cells (Fig. 3f), meanwhile, group 4 showed folding of the mucosal surface lining the secondary bronchi due to hyperplasia of the goblet cells with mucous exudate covering (Fig. 3g). At 7 dpi, in group 4, there was the formation of syncytial cells mixed with mononuclear cells in the lumen of the parabronchus (Fig. 3h), and many parabronchi of the same group showed massive infiltration of mononuclear and heterophilic cells that lead to occlusion of the parabronchi lumina (Fig. 3i) with the expression of the viral antigen in the inflammatory cell's infiltration (Fig. 3j).

\section{Proventriculus}

Gross examination of the proventriculus gland revealed focal ulceration in the mucosal surface of group 4 at 1 dpi. Petechial haemorrhages on the tips of the glands were noticed only in groups 3 and 4 at 5 dpi (Fig. 4a).

Group 1 preserved its normal histological structure among different sacrifices. Group 2 showed mild submucosal infiltration with mononuclear cells from 1 to $3 \mathrm{dpi}$, and multifocally infiltrating the necrosed glandular acini at 5 dpi (Fig. 4b). Meanwhile, groups 3 
Exploratory Animal and Medical Research, Vol.11, Issue 1, June, 2021

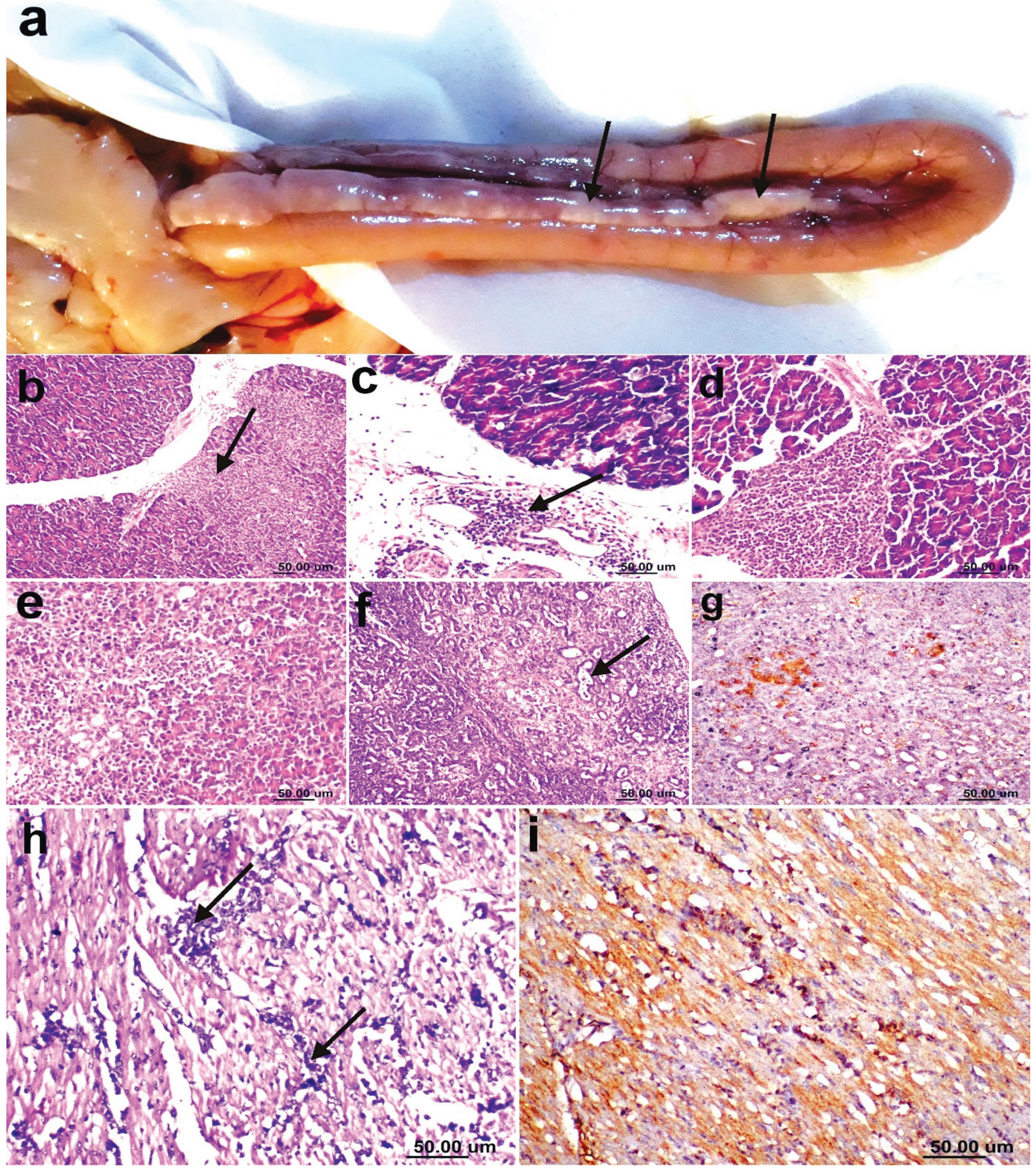

Fig. 6. Pathological findings of the pancreas and heart.

[(a) Group 3 at 3 dpi showing multifocal to diffuse pale necrotic zones (arrows). (b) Group 4 at 5 dpi presenting diffuse mononuclear cell infiltration (arrow) (H\&E). (c) Group 2 at 5 dpi showing peri-pancreatitis with diffuse mononuclear cells infiltration (arrow) (H\&E). (d) Group 2 at 7 dpi showing focal pancreatitis (H\&E). (e) Group 2 at 7 dpi showing severe necrosis of the exocrine acini (H\&E). (f) Group 4 at 7 dpi showing diffuse mononuclear cells infiltration and replacement of the acini by ductular structures separated by sparse amount of fibrous stroma(H\&E).(g) Group 4 at 7 dpi showing multifocal expression of the viral antigen in the necrosed pancreatic parenchyma (IHC). (h) Group 4 at 7 dpi showing disruption of the cardiac myofibers with a multifocal aggregation of mononuclear cells (arrows) (H\&E). (i) Group 4 at 7 dpi showing expression of the viral antigen in the disrupted cardiac myofibers and in the mononuclear cells infiltration (IHC)]. 
Pathological monitoring of visceral lesions induced by velogenic newcastle disease...

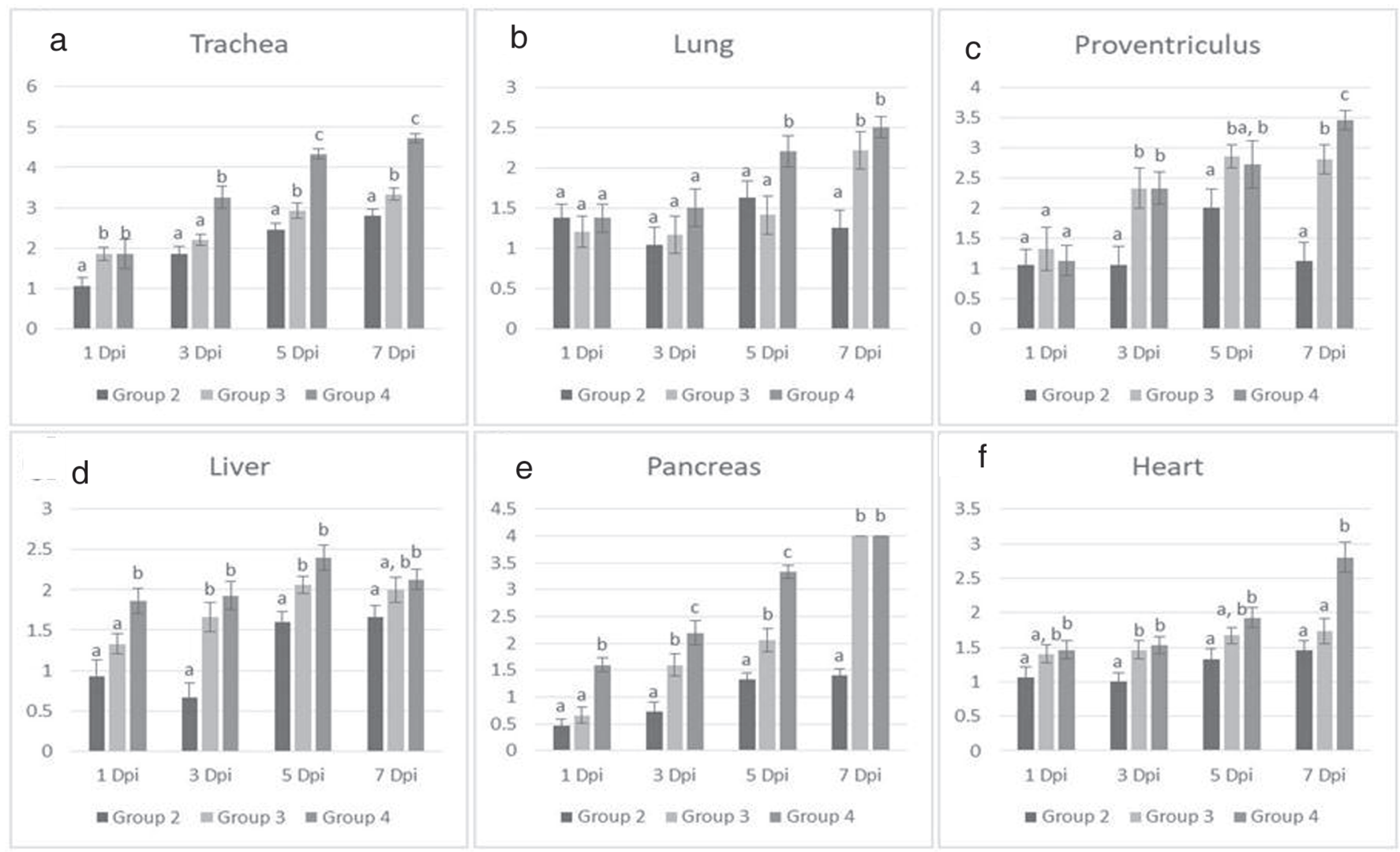

Fig. 7. Histopathological lesions score in the collected organs between different infected groups at 1, 3, 5 and 7 dpi.

[Values expressed as means \pm SE. Control group (group 1) scored 0 for histopathological alterations. Different letters (a-c) on the bars indicate statistically significant differences at $\mathrm{p}=0.05]$.

and 4 exhibited diffuse lymphoplasmacytic proventriculitis that characterized by submucosal infiltration with mononuclear cells from 3 to $5 \mathrm{dpi}$ meanwhile (Fig. 4c). Group 4 showed severe necrohemorrhagic proventriculitis at $5 \mathrm{dpi}$, in which the lumen of the glandular duct was filled with heterophils and fibrin (Fig. 4d), and the viral antigen was heavily expressed in the necrosed glands (Fig. 4e). At $7 \mathrm{dpi}$, fibroplasia of the lamina propria was observed in the mucosal layer of groups 3 and 4 (Fig. 4f). Also, catarrhal proventriculitis was detected in different infected groups with activation of mucous glands (Fig. 4g), moreover, group 4 showed severe depletion with eosinophilic cellular and karyorrhectic debris (lymphocytolysis) in the esophageal tonsil located in the lamina propria of the esophagus at the junction with the proventriculus (Fig. 4h).

\section{Liver}

No gross lesions were detected in the liver of all infected groups at 1 and 3 dpi. Meanwhile, many birds from group 4 exhibited greenish discoloration of the liver at 5 dpi and 7 dpi (Fig. 5a).

Microscopically, group 1 showed normal hepatic plates of two cells thick. Regarding the histopathological examination of the infected groups; group 2 showed slight vacuolation of the hepatocytes with sporadic hepatocellular necrosis among different sacrifices and were more prominent at 5 and 7 dpi. Groups 3 and 4 presented portal hepatitis which was characterized by portal mononuclear cell infiltration starting from $1 \mathrm{dpi}$ (Fig. 5b), with adjacent diffuse hepatocellular vacuolation noticed at 5 dpi (Fig. 5c). Additionally, inflammation was extended toward the necrosed hepatic plates in the birds from groups 3 and 4 (Fig. 5d). Liver sections from group 4 presented diffuse hepatic necrosis with mononuclear cells infiltration at 5 and $7 \mathrm{dpi}$ (Fig. 5e). Moreover, cholestasis was observed characterized by the accumulation of bile secretion in the bile ductules adjacent to the necrosed hepatic parenchyma (Fig. 5f). ND viral antigen expression was detected in the mononuclear cells infiltrating necrosed hepatic tissue of different infected groups from 5 to 7 dpi (Fig. 5 g).

\section{Pancreas}

Macroscopic examination of group 2 revealed no gross lesions during the experimental period. Meanwhile, groups 3 and 4 showed multifocal to diffuse pale necrotic 
zones at 5 and 7 dpi (Fig. 6a).

Group 1 showed normal histological structure with acinar epithelium that appeared roughly triangular and had deeply eosinophilic cytoplasm and large round nuclei. At 1 dpi, pancreatic tissue of all infected groups showed congestion with perivascular edema, in addition to focal lymphocytic infiltration in groups 3 and 4 . From 3 to 5 dpi, multifocal areas of mononuclear cells infiltration were detected in different infected groups, however, group 4 presented wider zones of infiltration (Fig. 6b) with numerous apoptotic exocrine acinar epithelium and hyperplasia of the intralobular ducts. Peripancreatitis was noticed in all infected groups from 3 to 7 dpi (Fig. 6c).

At 7 dpi, group 2 presented focal pancreatitis (Fig. 6d), meanwhile groups 3 and 4 demonstrated severe pancreatitis characterized by severe necrosis of the acini (Fig. 6e), with diffuse mononuclear cells infiltration and replacement of the acini by ductular structure separated by sparse amount of fibrous stroma (Fig. 6f). Viral antigen expression was detected mainly in groups 3 and 4 at 7 dpi (Fig. 6g).

In the present study, mononuclear inflammatory cells infiltration and hepatocellular degeneration and necrosis were detected. Similar findings were mentioned by Etriwati et al (2017) and Al-Azawy et al. (2018) detected immune-positive reaction for NDV in the macrophages and hepatocytes.

In agreement with our results, the lesions in the pancreas had been previously reported in NDV affected chickens (Wakamatsu et al. 2006, Susta et al. 2011, Anis et al. 2012).

As an explanation of the detected peripancreatic lesions induced by NDV, El-Bahrawy et al. (2015) stated that viral replication induced inflammatory reaction in the peripancreatic tissues and then on the pancreas. The virus most likely reached the peripancreatic tissues and pancreas at the same time by viremia, but infiltrating CD3-positive cells were detected in the peripancreatic tissues as early as 12 hours post-inoculation (hpi) and these cells started to infiltrate the adjacent pancreatic tissue from this time. The infiltration extended within the pancreas, eventually invading the entire exocrine pancreas. Moreover, NDV-nucleoprotein signals were detected in macrophages and other lymphoid cells at 1 dpi in the peripancreatic tissue were then observed in the pancreas by 5 dpi. Moreover, NDV-nucleoprotein signals were detected in macrophages and other lymphoid cells at 1 dpi in the peripancreatic tissue and then observed in the pancreas by 5 dpi.

In vivo studies support the role of macrophages and other lymphoid cells in NDV replication and further dissemination to other tissues (Brown et al. 1999, Kommers et al. 2003, Lu et al. 2014). The detected damage in the exocrine tissue of the pancreas after NDV infection may be attributed to the protease content in the exocrine cells, which may enhance viral replication (ElBahrawy et al. 2015).

\section{Heart}

Capillary congestion with few mononuclear cells was detected in all infected groups at 1 and 3 dpi. At 5 dpi, degeneration of myocardial fibers was noticed mainly in group 4 and less commonly in group 3 . At 7 dpi, group 3 showed pericarditis with individual necrosis of cardiac myocytes, while group 4 showed disruption of the cardiac myofibrils with a multifocal aggregation of mononuclear cells (Fig. 6h), in which expression of the viral antigen was detected (Fig. 6i).

Epicarditis and myocarditis with myocardium degeneration, and necrosis, edema, with mononuclear cell infiltration as well as the immune-positive reaction detected in the cytoplasm of the myocardium, inflammatory cell, and in vascular endothelial cell were also mentioned by Etriwati et al. (2017) due to NDV infection.

\section{Histopathological lesion score}

Histopathological lesions score of the different experimental groups has been shown in Fig.7 including different examined organs at 1, 3, 5, 7 dpi.

The tracheal lesion score at 1 and 3 dpi showed a nonsignificant difference between groups ( 3 and 4 ) and groups ( 2 and 3 ) respectively. While lung lesion score showed no significant difference between the three infected groups from 1 to 3 dpi (Fig. 7a and 7b).

However, from 5 to $7 \mathrm{dpi}$ a significant difference was detected among the different infected groups with the highest pulmonary lesion score in group 4 reaching 4.33 and 4.73 respectively. Also, the lung of group 4 recorded the highest lesions score from 5 to 7 dpi reaching 2.21 and 2.50 respectively.

Concerning the proventriculus, groups 3 and 4 showed no significant difference from 1 to $5 \mathrm{dpi}$, however, at 7 dpi, a significant difference was found between all infected groups in which group 4 recorded the peak at 3.46 (Fig. 7c). Regarding the liver, group 2 recorded the least lesions score among different birds compared with the birds of groups 3 and 4 . A significant difference was detected between groups 3 and 4 at $1 \mathrm{dpi}$, conversely, at 3, 5, and $7 \mathrm{dpi}$, no significant difference was detected between the above-mentioned groups (Fig. 7d).

Referring to the pancreas, at $1 \mathrm{dpi}$, a significant 
Pathological monitoring of visceral lesions induced by velogenic newcastle disease...

difference in pancreatic lesion score was observed between group 4 and the other infected groups. From 3 to $5 \mathrm{dpi}$, a significant difference was found among all infected groups, in which, group 4 presented the highest lesion score; 2.20 and 3.33 respectively. At $7 \mathrm{dpi}$, groups 3 and 4 showed the maximum lesions score (4.00) (Fig. 7e).

Statistical analysis of lesion score of the heart showed no significant difference between groups 2 and 3 at 1, 5, and $7 \mathrm{dpi}$, and between groups 3 and 4 from 1 to $5 \mathrm{dpi}$.

At 7 dpi, a significant difference in cardiac lesion score was observed between group 4 and the other infected groups (Fig. 7f).

Newcastle disease is regarded as a significant pathogen for chicks. The virus can infect a large number of avian species (Pansota et al. 2013). ND is mostly caused by velogenic strains of NDV than mesogenic or lentogenic strains (Pansota et al. 2013). Velogenic strain of ND virus may cause $100 \%$ mortality (Alexander 1997).

In agreement with Etriwati et al. (2017), the confirmation of NDV residence in tissue by immunohistochemical staining proves that the immunohistochemistry method can be used as an accurate, quick, and more economical method than serological and molecular methods.

Using quantitative real-time RT-PCR to isolate viral RNA from lungs tissue, demonstrated the increase of viral load in lung tissue with advancing age of the bird supporting our point of view regarding the higher susceptibility of older chicken to VNDV. Our findings regarding the esophageal tonsils included haemorrhages and ulceration at the site of lymphoid aggregate are consistent with those of Cattoli et al. (2011), these findings could be due to the necrosis of lymphocytes induced by the virus (Onyema et al. 2019).

\section{CONCLUSION}

The present work showed age related difference in the pathological picture of NDV in experimentally infected broiler chickens and reflected the influence of age on virus virulence. There was an age related increase in pulmonary expression of IL- 6 and IFN- $\gamma$ with subsequent related pulmonary pathology. The intraocular route induced systemic dissemination of virus that confirmed (by immunohistochemistry) residence of virus antigen in different organs.

\section{REFERENCES}

Ahmed AI, Odisho SM (2018) Comparison of the immune response between local manufactured and commercial inactivated Newcastle Disease Virus vaccine in a challenge trail with field isolated Newcastle Disease Virus. Iraqi J Vet Med 2: 46-51.

Al-Azawy AK, Al-Ajeeli KS, Ismail A (2018) Isolation and Identification of wild Isolate of Newcastle Disease Virus from broiler farm in Diyala province: virological and histopathological study. Iraqi J Vet Med 42: 42-50.

Alexander DJ (1997) Newcastle disease and other avian paramyxoviridae infections. 541-569. In: Calnek BW, Barness HJ, Beard CW, McDuogald LR, Saif YM (eds.), Diseases of poultry, $10^{\text {th }}$ edn., Iowa State University Press, Ames, Iowa, USA.

Alexander DJ (2003) Newcastle disease and other avian paramyxoviridae infections. In: Diseases of poultry, eds. Saif YM, Barnes HJ, Glisson JR, Fadly AM, McDougald LR, Swayne DE, $11^{\text {th }}$. edn., Iowa State University Press, Ames, IA,. Iowa State Univ Press Ames, IA. 64-87.

Anis Z, Morita T, Azuma K, Ito H, Ito T, Shimada A (2012) Comparative study on the pathogenesis of the generated $9 \mathrm{a} 5 \mathrm{~b}$ Newcastle disease virus mutant isolate between chickens and waterfowl. Vet Pathol 50: 638-647.

Bancroft JD (2013) Histochemical Techniques. Imprints: Butterworth-Heinemann. $2^{\text {nd. }}$ edn, ISBN: 9780407000339.

Barton JT, Bickford AA, Cooper GL, Charlton BR, Cardona CJ (1992) Avian Paramyxovirus type 1 infections in racing pigeons in California. I. Clinical signs, pathology, and serology. Avian Dis : 463-468.

Brown C, King DJ, Seal BS (1999) Pathogenesis of Newcastle disease in chickens experimentally infected with viruses of different virulence. Vet Pathol 36:125-132.

Burns, Robert, Ed. (2005) Immunochemical protocols. Vol 295 Totowa, NJ Humana Press.

Cattoli G, Susta L, Terregino C, Brown C (2011) Newcastle disease a review of field recognition and current methods of laboratory detection. J Vet Diagnostic Investig 23: 637-656.

Chukwudi OE, Chukwuemeka ED, Mary U (2012) Newcastle disease virus shedding among healthy commercial chickens and its epidemiological importance. Pak Vet J 32: 354356.

Desingu PA, Singh SD, Dhama K, Kumar ORV, Malik YS, Singh R (2017) Clinicopathological characterization of experimental infection in chickens with sub-genotype VIIi Newcastle disease virus isolated from peafowl. Microb Pathog 105: 8-12. 
Exploratory Animal and Medical Research, Vol.11, Issue 1, June, 2021

Dortmans JC, Koch G, Rottier PJ, Peeters BP (2011) Virulence of newcastle disease virus: What is known so far? Vet Res 42: 1-11.

El-Bahrawy A, Zaid A, Sunden Y, Sakurai M, Ito H et al. (2015) Pathogenesis of pancreatitis in chickens after experimental infection with 9a5b Newcastle disease virus mutant isolate. J Comp Pathol 153: 315-323.

Etriwati, Ratih D, Handharyani E, Setiyaningsih S (2017) Pathology and immunohistochemistry study of Newcastle disease field case in chicken in Indonesia. Vet World 10: 10661071.

Gotoh B, Ohnishi Y, Inocencio NM, Esaki E, Nakayama K et al. (1992) Mammalian subtilisin-related proteinases in cleavage activation of the paramyxovirus fusion glycoprotein: superiority of furin/PACE to PC2 or PC1/PC3. J Virol 66: 63916397.

Hanson RP, Spalatin J, Jacobson GS (1973) The viscerotropic pathotype of Newcastle disease virus. Avian Dis 17: 354-361.

Hirano T (1998) Interleukin 6. In: AE Thomson (ed.).The Cytokine Handbook 3rd edition. Academic Press San Diego. 197-227.

Hussein EA, Hair-Bejo M, Adamu L, Omar AR, Arshad SS et al. (2018) Scoring system for lesions induced by different strains of Newcastle disease virus in chicken. Vet Med Int 2018: $1-9$.

Iroegbu CU, Emeruwa AC (1993) Recovery of velogenic Newcastle disease virus from dead and healthy free-roaming birds in Nigeria. Avian Pathol 22: 383-387.

Kaiser P, Rothwell L, Galyov EE, Barrow PA, Burnside J, Wigley P (2000) Differential cytokine expression in avian cells in response to invasion by Salmonella typhimurium, Salmonella enteritidis and Salmonella gallinarum. Microbiology 146: 3217-3226.

Kim SH, Xiao S, Shive H, Collins PL, Samal SK (2012) Replication, neurotropism, and pathogenicity of avian paramyxovirus serotypes 1-9 in chickens and ducks. PLoS One 7: e34927.

Kommers GD, King DJ, Seal BS, Brown CC, King ADJ et al. (2003) Pathogenesis of chicken-passaged Newcastle disease viruses isolated from chickens and wild and exotic birds. Avian Dis 47: 319-329.

Kommers GD, King J, Seal BS, Carmichael KP, Bown CC (2002) Pathogenesis of six pigeon-origin isolates of Newcastle disease virus for domestic chickens. Vet Pathol 39: 353-362.
Kothlow S, Kaspers B (2008) The avian respiratory immune system. IN: Davison F, Kaspers B, Schat KA (eds.) Avian Immunol, $1^{\text {st }}$. edn. Acad Press Elsevier, San Diego : 273-288.

Liu XF, Wan HQ, Ni XX, Wu YT, Liu WB (2003) Pathotypical and genotypical characterization of strains of Newcastle disease virus isolated from outbreaks in chicken and goose flocks in some regions of China during 1985-2001. Arch Virol 148: 1387-1403.

Livak KJ, Schmittgen TD (2001) Analysis of relative gene expression data using real-time quantitative PCR and the 2$\Delta \Delta \mathrm{CT}$ method. Methods 25: 402-408.

Lu A, Diao Y, Chen H, Wang J, Ge P et al. (2014) Evaluation of histopathological changes, viral load and immune function of domestic geese infected with Newcastle disease virus. Avian Pathol 43: 325-332.

Manzoor AW, Rizvi F, Javed M, Numan M, Khan A, Rehman SU (2013) Pathotyping of Newcastle disease virus using multiplex reverse transcription polymerase chain reaction and pathological studies in naturally infected broiler chicks. Pak J Life Soc Sci 11: 225-232.

De Mayer EM, De Mayer-Guinard J (1998) Interferons. In: Thomson AW (editor). The cytokine handbook $3^{\text {rd }}$ edn. Acad Press San Diego : 491-515.

Mayo MA (2002) A summary of taxonomic changes recently approved by ICTV. Arch Virol 147: 1655-1656.

Mohammed FF, Mousa MR, Khalefa HS, El-Deeb AH, Ahmed KA (2019) New insights on neuropathological lesions progression with special emphasis on residence of velogenic newcastle disease viral antigen in the nervous system of experimentally infected broiler chickens. Explor Anim Med Res 9(2): 145-157.

Mousa MR, Mohammed FF, Khalefah HS, El-deeb AH, Ahmed KA (2019) Comparative serological, histopathological and immunohistochemical evaluation of immune status of broiler chickens experimentally infected with velogenic Newcastle disease virus in different ages. Inter J Vet Sci 8: $143-150$.

Mousa MR, Mohammed FF, Reheem FAA, El-deeb AH, Ahmed KA (2020) Characterization of renal inflammatory cytokines and related nephropathy in experimentally infected broiler chickens with velogenic Newcastle disease virus. J World's Poult Res 10(1): 109-117.

Mousa MR, Mohammed FF, El-Deeb AH, Khalefa HS, Ahmed KA (2020) Molecular and pathological characterisation 
Pathological monitoring of visceral lesions induced by velogenic newcastle disease...

of genotype VII Newcastle disease virus on Egyptian chicken farms during 2016-2018. Acta Vet Hungarica 68(2): 221-230.

Nagai Y (1995) Virus activation by host proteinases. a pivotal role in the spread of infection, tissue tropism and pathogenicity. Microbiol Immunol 39: 1-9.

Nakamura K, Yamada M, Yamaguchi S, Mase M, Narita M et al. (2001) Proliferation of lung macrophages in acute fatal viral infections in chickens. Avian Dis. 45: 813-818.

OIE (2012) Newcastle disease. Manual of Diagnostic Tests and Vaccines for Terrestrial Animals. 555-574.

Okamoto K, Ohgimoto S, Nishio M, Tsurudome M, Kawano M et al. (1997) Paramyxovirus-induced syncytium cell formation is suppressed by a dominant negative fusion regulatory protein-1 (FRP-1)/CD98 mutated construct: an important role of FRP-1 in virus-induced cell fusion. J Gen Virol 78: 775-783.

Oladele SB, Enoch I, Ibrahim NDG (2008) Changes in histopathology, haematocrit, haemoglobin, haemagglutination inhibition antibody titre and total protein of Japanese quails (Coturnix coturnix japonica) administered different doses of Newcastle disease virus. J Anim Vet Adv 7(4): 418-424.

Onyema I, Eze DC, Abba Y, Emennaa PE, Shoyinka SVO et al. (2019) Lesions of velogenic viscerotropic Newcastle disease virus infection were more severe in broilers than pullets. J Appl Anim Res 47:1 89-194.

Pansota FM, Rizvi F, Sharif A, Javad MT, Muhammad G et al. (2013) Use of hy perimmune serum for passive immunization of chicks experimentally infected with Newcastle disease virus. Pak J Agri Sci 50: 279-288.

Panus A, Setiyaningsih S, Mayasari NLPI (2015) Newcastle disease virus infection study on duck and chicken in Subang district. J Ilmu Ternak dan Vet 20: 134-147.

Piacenti M, King DJ, Seal BS, Zhang J, Brown CC (2006) Pathogenesis of Newcastle disease in commercial and specific pathogen-free turkeys experimentally infected with isolates of different virulence. Vet Pathol 43: 168-178.
Reed LJ, Muench H (1938) A simple method of estimating fifty per cent endpoints. Am J Epidemiol 27: 493-497.

Rue CA, Susta L, Cornax I, Brown CC, Kapczynski DR et al. (2011) Virulent Newcastle disease virus elicits a strong innate immune response in chickens. J Gen Virol 92: 931-939.

Samiullah M, Rizvi F, Anjum AD, Shah MFA (2006) Rising hyperimmune serum against avian paramyxovirus (APMV-1) and pigeon paramyxovirus (PPMV-1) in rabbits and their crossreactivity. Pak J Biol Sci 9: 2184-2186.

Shahzad M, Rizvi F, Khan A, Siddique M, Zargham Khan M, Bukhari SM (2011) Diagnosis of avain paramyxovirus Type1 infection in chicken by immunoflourescence technique. Int $\mathbf{J}$ Agric Biol 13: 266-270.

Siddique AB, Rahman SU, Hussain I, Muhammad G (2012) Frequency distribution of opportunistic avian pathogens in respiratory distress cases of poultry. Pak Vet J 32: 386-389.

Susta L, Miller PJ, Afonso CL, Brown CC (2011) Clinicopathological characterization in poultry of three strains of Newcastle disease virus isolated from recent outbreaks. Vet Pathol 48: 349-360.

Wakamatsu N, King DJ, Kapczynski DR, Seal BS, Brown CC (2006) Experimental pathogenesis for chickens, turkeys, and pigeons of exotic Newcastle disease virus from an outbreak in California during 2002-2003. Vet Pathol 43: 925-933.

Wigley P, Kaiser P (2003) Avian cytokines in health and disease. Brazilian J Poult Sci 5: 1-14.

Wise MG, Suarez DL, Seal BS, Pedersen JC, Senne DA et al. (2004) Development of a real-time reverse-transcription PCR for detection of Newcastle disease virus RNA in clinical samples. J Clin Microbiol 42: 329-338.

Xiao S, Paldurai A, Nayak B, Samuel A, Bharoto EE et al. (2012) Complete genome sequences of Newcastle disease virus strains circulating in chicken populations of Indonesia. J Virol 86: 5969-5970.

*Cite this article as: Mousa MR, Mohammed FF, El-Deeb AH, AmerF, Ahmed KA (2021) Pathological monitoring of visceral lesions induced by velogenic Newcastle disease in experimentally infected broiler chickens at different ages with special reference to pulmonary inflammatory cytokines expression. Explor Anim Med Res 11(1): 67-79. DOI : $10.52635 / E A M R / 11.1 .67-79$ 